Itinéraires Itinéraires

Littérature, textes, cultures

2013-2 | 2014

Sade et les femmes

\title{
Sade et la réécriture de soi dans « Eugénie de Franval »
}

Vincent Jolivet

\section{(2) OpenEdition}

\section{Journals}

Édition électronique

URL : http://journals.openedition.org/itineraires/702

DOI : 10.4000/itineraires.702

ISSN : 2427-920X

Éditeur

Pléiade

\section{Édition imprimée}

Date de publication : 1 mars 2014

Pagination : 101-112

ISBN : 978-2-343-02712-8

ISSN : $2100-1340$

\section{Référence électronique}

Vincent Jolivet, « Sade et la réécriture de soi dans « Eugénie de Franval » », Itinéraires [En ligne], 2013-2 | 2014, mis en ligne le 01 novembre 2013, consulté le 04 mai 2019. URL : http:// journals.openedition.org/itineraires/702 ; DOI : 10.4000/itineraires.702

\section{(ब) $(\Theta \Theta$}

Itinéraires est mis à disposition selon les termes de la licence Creative Commons Attribution - Pas d'Utilisation Commerciale - Pas de Modification 4.0 International. 


\section{Sade et la réécriture de soi dans « Eugénie de Franval»}

\section{Résumé}

Si Sade renonça finalement à écrire ses mémoires, projet qu'il caressa un temps, la critique contemporaine a montré que son œuvre littéraire a pu, pour une part, jouer ce rôle pour lui, rejouant via l'écriture des moments marquants de sa vie ou réfractant par le biais de personnages contrastés les contradictions d'une personnalité plus clivée qu'il n'y paraissait. Centré sur « Eugénie de Franval », le présent article s'emploie donc à montrer comment cette nouvelle, issue des Crimes de l'amour, relève dans une large mesure d'une inspiration autobiographique. Les principaux personnages y font en effet figure de doubles de l'écrivain et de ses proches, et la trame des événements qui vient les unir rappelle étrangement le déroulement de la liaison scandaleuse entretenue par le marquis avec sa belle-sœur Anne-Prospère de Launay. Dans cette réécriture de sa propre destinée, Sade entreprend ainsi de hisser ses amours incestueuses à la hauteur de drame mythique de la passion, tout comme il s'efforce de percer les mystères de son incarcération et d'en exorciser la souffrance, au fil de revanches symboliques et de repentirs ambigus.

Mots clés : réécriture de soi, fonctions de l'écriture, recomposition littéraire des événements, « Eugénie de Franval », affaire de Marseille

\section{Abstract}

There was a time in his life when Sade considered the idea of writing his memoires. Although he finally never did so, critics have shown that his novels reflect some parts of his personality or hint at some precise events of his life. This article, which focuses on "Eugénie de Franval," aims at showing how this short story can be considered as autobiographic to some extent. Indeed, the main characters resemble Sade and his female relatives quite significantly, and the related events strongly echo with the scandalous affair he had with his stepsister Anne-Prospère de Launay and its dramatic outcome. Rewriting his own story, Sade attempts to turn his lost love into a mythical story of incestuous passion, takes pleasure in getting symbolic revenges on those who hurt him in the past, and also tries to achieve a better comprehension of the circumstances leading to his imprisonment.

Keywords: self rewriting, writing functions, life literary recomposing, "Eugénie de Franval", case of Marseille 
L'œuvre sadienne appelle l'identification de son auteur aux personnages qu'elle met en scène. Dès la parution de Justine ou les Malheurs de la vertu (1791), une logique de va-et-vient de l'œuvre à l'homme, de l'homme à l'œuvre, se met en place dans le public : la monstruosité criminelle du libertin s'y enrichit du surcroît d'effroi né des rumeurs entourant le marquis, le mythe sadien s'y colore à l'inverse de la substance de ses romans. Les silhouettes respectives de l'écrivain maudit et du scélérat né de ses fantasmes en viennent alors à se confondre dans l'imaginaire populaire, assimilation nourrie de peurs collectives appelée à perdurer pendant près d'un siècle et demi. C'est sans doute le mérite des biographes d'avoir su dénouer cet écheveau de racontars et de légendes colportés à peu de frais; c'est aussi celui d'une certaine critique d'avoir montré, par-delà les évidences et les raccourcis simplistes, la profondeur et la complexité du rapport entretenu par Sade avec ses créatures d'encre et de plume. On doit ainsi à Jean Paulhan la mise en évidence de la paradoxale identification du marquis à ses personnages de victimes - car, qu'on le veuille ou non, Sade est au moins autant à chercher dans la chair frémissante de Justine que dans la rage destructrice de ses bourreaux ${ }^{1}-$, intuition essentielle qu'il revint ensuite à Michel Delon d'approfondir, pour proposer de l'œuvre une lecture attentive aux résonances biographiques ${ }^{2}$, sensible aux recompositions intimes. Véritable autoportrait en miroir brisé, celle-ci fait voler en éclats le cadre unifié des mémoires ou de la confession pour offrir une succession d'éclairages romanesques, parcellaires et contradictoires, où se profilent fugitivement les traits d'un visage sans cesse dérobé. Nombre de personnages y font figure de doubles fictionnels de l'écrivain, présentent avec lui une certaine ressemblance, trop marquée pour être anodine, trop circonscrite pour prétendre épuiser la vérité de leur modèle : s'il appartient ainsi au marquis de Bressac de systématiser l'homosexualité de Sade, d'en magnifier les rapports sodomites avec le valet Latour, le comte de Gernande vient pour sa part réfracter dans sa criminelle boulimie l'obésité qui gagne un Sade privé de mouvement comme de liberté; si le chirurgien Rodin permet de compléter cette image criminelle en endossant le costume inquiétant du vivisecteur fantasmé par le public, le personnage de Valcour participe au contraire d'un dessein auto-apologétique et témoigne d'une aspiration à la rédemption.

1. Jean Paulhan, «La douteuse Justine, ou les revanches de la pudeur », préface aux Infortunes de la vertu, Paris, Gallimard, coll. « Folio », 1970.

2. Michel Delon, «Sade autobiographe : les personnages de Valcour et de Rodin », dans Mary Donaldson-Evans, Lucienne Frappier-Mazur et Gerald Prince (dir.), Autobiography, Historiography, Rhetoric. A Festschrift in honor of Frank Paul Bowman, Amsterdam, Rodopi, 1994, p. 75-86. Bressac, Rodin, Gernande appartiennent tous au cycle de Justine. Valcour est, lui, le héros éponyme du « roman philosophique » du marquis, Aline et Valcour (1795). 
C'est dans la lignée de ces travaux fondateurs que nous souhaiterions inscrire notre étude, pour proposer d'un texte encore à ce jour trop peu étudié ${ }^{3}$, "Eugénie de Franval», une lecture soucieuse d'en explorer la part autobiographique ${ }^{4}$. Il s'agirait d'y montrer l'ampleur des emprunts de l'écrivain à sa propre existence, l'inscription dans le cœur de la fiction de souvenirs brûlants - ceux qui l'unissent au grand trio féminin qui marqua dramatiquement sa vie : sa femme, Renée-Pélagie; la sœur de celle-ci, Anne-Prospère de Launay; et leur mère, la présidente de Montreuil. Il s'agirait aussi de mettre en lumière et d'analyser les déformations que Sade fait subir à ce matériau biographique, d'aller chercher la vérité de l'être dans la réécriture de soi, ce subtil jeu de déplacements qui réagence les faits et les souvenirs, où s'élabore un autre moi tissé de réel et d'imaginaire. Dans ce cadre, on le sent, l'écriture autobiographique se définirait moins comme le plat relevé des événements passés, que comme la trame hautement subjective des faits et des fantasmes, la somme des désirs et des frustrations, des rancœurs et des nostalgies; autobiographie qui ne s'avoue pas, donc, et pour une grande part inconsciente; autobiographie cryptée, pour finir, qui n'offre à son lecteur qu'un réseau ténu d'indices incertains pour s'aventurer dans le labyrinthe du texte.

Petit bijou tiré du recueil des Crimes de l'amour (1800), « Eugénie de Franval » fait figure d'exception dans l'œuvre du marquis de Sade : là où les malheurs de Justine semblent voués à l'amplification infinie - chaque nouvelle version surenchérissant sur celle qui la précède -, la course romanesque d'Eugénie brille au contraire par sa condensation comme par sa brièveté; là où les textes libertins ne cessent de dénoncer l'illusion amoureuse, l'immoralisme incestueux prévalant ici sait à l'inverse se parer des prestiges les plus purs de la passion; là, enfin, où les écrits clandestins déploient le crime dans sa liberté triomphante, l'œuvre exotérique lui confère plutôt l'éclat ambigu d'une destinée tragique. À lire les quelques dizaines de pages que compte la nouvelle, l'identité de son auteur ne saurait cependant guère faire l'objet d'un doute, tant s'y donnent libre cours divers traits caractéristiques de l'écriture sadienne : intégration à la fiction d'un

3. Voir tout de même la belle préface de Jean Fabre aux Crimes de l'amour, qui fait d'« Eugénie de Franval» le chef-d'œuvre méconnu de Sade (Euvres complètes, éd. Gilbert Lely, Paris, Cercle du livre précieux, 1966-1967, t. X, p. XI-XXXVI), et les analyses de Christophe Martin, qui confronte l'éducation négative mise en place par Franval à celle imaginée par Rousseau dans l'Émile (Éducations négatives : fictions d'expérimentation pédagogique au dix-huitième siècle, Paris, Classiques Garnier, 2010, p. 273-282).

4. Il est à noter que la dimension autobiographique de cette nouvelle n'avait pas échappé à Pierre Klossowski dans la préface qu'il consacre lui aussi aux Crimes de l'amour dans l'édition des Euvres complètes : «Le caractère de Franval et celui de son épouse ne sont pas sans éléments autobiographiques. Mme de Franval a tous les traits, toutes les vertus d'amour, de dévouement et de résignation de la marquise à l'égard du caractère récidiviste du marquis. De son côté, Franval, plus encore que d'autres personnages, offre toute l'irréfrénable impulsion de Sade » (op. cit., p. XLV-XLVI). 
certain dialogisme philosophique ${ }^{5}$, goût pour la mise en scène de véritables tableaux luxurieux ou pathétiques ${ }^{6}$, présence de motifs et de faits de styles chers à l'écrivain ${ }^{7} .$. Mais au-delà de cet ensemble d'éléments, qui donnent au texte sa tonalité proprement sadienne et l'intègrent au vaste ensemble de l'œuvre, la nouvelle signe aussi son origine dans les nombreuses ressemblances qu'elle présente avec ce que l'on sait de l'existence tumultueuse du marquis. On est ainsi frappé par la répartition des rôles à l'intérieur de la nouvelle, qui semble reconduire, à l'identique ou presque, les structures affectives et familiales qui donnèrent à la vie de l'écrivain la forme qu'on connait. On ne peut ignorer non plus la troublante proximité des destins, qui bien souvent fait épouser aux personnages de la fiction la trajectoire douloureuse de leurs modèles supposés. Dans les échos biographiques semés au fil des pages se suggère toujours un peu plus le visage de celui qui tient la plume; sous le prétexte opportun de la «nouvelle tragique» s'élabore la recomposition littéraire d'un tissu de situations vécues.

Jeune, séduisant, mais profondément libertin, le personnage central de la nouvelle, Franval, fait inévitablement penser au Sade qui n'a pas encore connu l'épreuve des prisons, dont il constitue sans nul doute une sorte de double idéalisé. Il partage en effet avec son créateur le goût immodéré pour la débauche, l'appartenance à la plus haute noblesse et la tendance à infléchir le matérialisme athée dans le sens d'un relativisme criminel. Comme lui, il se montre « plein de mépris pour les devoirs moraux et religieux ${ }^{8} »$; comme lui, il souffre en premier lieu d'une imagination ardente, qui lui fait concevoir tous les excès :

Un désordre d'imagination, au-delà de tout ce qu'on peut peindre, était le premier défaut de Franval; on ne se corrige point de celui-là; la diminution des forces ajoute à ses effets; moins l'on peut, plus l'on entreprend; moins on agit, plus on invente; chaque âge amène de nouvelles idées, et la satiété, loin de refroidir, ne prépare que des raffinements plus funestes ${ }^{9}$.

Il n'est pas anodin de remarquer la proximité de ces analyses psychologiques avec certains propos de la correspondance, dans lesquels

5. Principalement lors de la discussion entre Franval et Clervil (« Eugénie de Franval », dans Les Crimes de l'amour, éd. Michel Delon, Paris, Gallimard, 1987, p. 334-339).

6. Voir, dans le premier cas de figure, la scène où Mme de Franval supplie à genoux sa fille de renoncer à son intrigue criminelle ou celle de son évanouissement à la suite des accusations malhonnêtes de son époux; voir, dans le second, le passage où Valmont contemple Eugénie dénudée (Ibid., p. 324, 332 et 348-349).

7. Pensons au motif récurrent dans la fiction sadienne de la haine de la mère ou à celui du matricide, sur lesquels nous reviendrons; pensons également à ce goût, typique chez Sade, pour l'énumération dans une même phrase des crimes dont se couvre un personnage : « séduite par vous, ma fille se rend à la fois coupable d'inceste, d'adultère, de libertinage et de l'ingratitude la plus odieuse envers celle qui l'a mise au monde... (Ibid., p. 327).

8. Ibid., p. 292.

9. Ibid. 
l'écrivain se plaint d'une détention qui lui échauffe le sang et vient aigrir son imagination ${ }^{10}$ : le portrait romanesque se nourrit ici de l'expérience carcérale du marquis, qui trouve à s'exprimer sous un jour plus serein que dans les lettres par le détour de la fiction. L'insistance sur la place du père libertin dans l'éducation reçue - " grand partisan des sophismes à la mode ", celui de Franval encourage son fils « à penser solidement sur toutes ces matières ${ }^{11} \gg$ et lui transmet des ouvrages qui hâtent sa corruption - peut pour sa part faire écho au rapport privilégié entretenu par Sade avec le sien; mais les correspondances familiales s'arrêtent là : si la mort précoce de ses parents laisse Franval richissime et maître de lui-même, celle du père du marquis livrait un fils désargenté à la tutelle d'une bellefamille plus fortunée. Une même logique d'embellissement de la réalité se trouve à l'œuvre dans la place ménagée à la figure de l'oncle : celui de la fiction meurt opportunément à la majorité de son neveu, non sans lui avoir entre-temps dûment remis sa succession et trouvé une épouse à sa convenance; celui de l'existence réelle, l'abbé d'Ébreuil, un temps proche du marquis, dont il encadra les premières années, se lassa très vite des foucades de celui-ci, contre lequel il en vint même à solliciter une lettre de cachet. La fiction préfère recueillir le souvenir des affections enfantines plutôt que celui des brouilles de l'âge mûr.

À côté de ce mari criminel, Mme de Franval ferait pâle figure, n'étaient la constance de son dévouement et le caractère exemplaire de sa mort. Par bien des traits, elle rappelle Renée-Pélagie : douce et dévote comme elle, elle épouse un homme qu'elle ne connaît guère, mais pour lequel elle éprouve très vite un amour indéfectible; comme elle, elle fait de celui-ci l'unique préoccupation de son existence :

Honnête, sensible, bien élevée et volant par amour au-devant des désirs du seul homme qui l'occupait au monde, Mme de Franval porta ses fers les premières années sans soupçonner son esclavage; il lui était aisé de voir qu'elle ne faisait que glaner dans le champ de l'hymen, mais trop heureuse encore de ce qu'on lui laissait, sa seule étude, son attention la plus exacte, était que, dans ces courts moments accordés à sa tendresse, Franval pût rencontrer au moins tout ce qu'elle croyait nécessaire à la félicité de cet époux chéri ${ }^{12}$.

Difficile de ne pas lire dans ces quelques lignes une évocation voilée de celle qui partagea la plupart des malheurs de Sade. Indulgente, à défaut

10. «Vous devez connaître assez mon cœur pour être persuadée que le soupçon seul du déshonneur est capable de le refondre en entier, et vous avez assez d'esprit pour comprendre qu'une faute dont l'origine est dans l'effervescence du sang ne se corrige pas en aigrissant encore plus ce sang, en allumant le cerveau par la retraite et enflammant l'imagination par la solitude » (Sade, «Lettre à la présidente de Montreuil du 13 mars 1777 », dans EEuvres complètes, éd. Gilbert Lely, Paris, Cercle du livre précieux, 1966-1967, t. XII, p. 118).

11. Sade, «Eugénie de Franval », op. cit., p. 292. Sade souligne.

12. Ibid., p. 295. 
d'y être insensible, aux torts d'un mari trop profondément aimé, Mme de Franval se prête naïvement à toutes les réconciliations. Plus belle, pour les besoins de la littérature, que son modèle original, elle partage cependant significativement avec lui une extraction sociale plus basse que celle de son époux ${ }^{13}$. Elle apparait socialement isolée, conformément aux exigences de Franval qui ne souhaitait de femme qu' « avec le moins d'entours possible ${ }^{14} »$, ce qui la livre au bon plaisir de son mari, quand c'est au contraire l'existence de Sade qui fut, dans l'ordre non imaginaire des choses, marquée par l'isolement au sein de sa caste - ce qui pour une part explique ses déboires judiciaires -, et l'étroite dépendance du réseau de relations de sa belle-famille.

Dans la nouvelle, celle-ci se résume à la seule figure de Mme de Farneille, dont on ne peut que noter, là encore, l'évidente ressemblance avec la propre belle-mère du marquis, si importante dans le cours de la vie de Sade. L'écrivain la décrit d'abord comme une femme encore jeune et belle, pleine d'intelligence et de charme - conforme, en somme, à ce que l'on peut savoir de son modèle ${ }^{15}-$; surtout, il lui fait jouer dans le cadre de sa nouvelle le rôle qu'il prête à la présidente de Montreuil dans son drame personnel ${ }^{16}$ : relativement discrète au commencement, la présence de Mme de Farneille ne cesse de s'affirmer tout au long d' « Eugénie de Franval », au point de faire d'elle la grande adversaire du héros criminel, celle qui, par ses interférences et ses machinations, vient faire basculer son destin. Sade se plaît d'abord à la peindre en belle-mère faible et impuissante : la littérature autorise ces revanches de papier, celles où viennent se panser les meurtrissures du moi et les blessures de dignité. Nul doute que Sade ait donc trouvé bien du plaisir à tracer ces lignes où s'inverse un rapport de force qui ne lui fut que trop défavorable dans la vie; où le détenu ruiné se rêve en grand seigneur libre d'emprisonner qui bon lui semble :

La belle-mère de Franval n'ayant plus de parents... peu de crédit par elle-même [...], ayant à lutter contre un gendre aimable, jeune, bien placé, s'imagina fort sensément qu'il était plus simple de s'en tenir à des représentations, que d'entreprendre des voies de rigueur, avec un homme qui ruinerait la mère et ferait enfermer la fille, si l'on osait se mesurer à lui; moyennant quoi, quelques remontrances furent tout ce qu'elle hasarda ${ }^{17}$.

Mais ce fantasme fait cependant long feu très vite, comme si l'écrivain était incapable d'imaginer durablement une belle-mère qui ne soit

13. Mme de Franval appartient par sa famille au monde de la finance, René Pélagie à celui de la noblesse de robe.

14. Sade, «Lettre à la présidente de Montreuil... », op. cit., p. 293.

15. Voir Jean-Jacques Pauvert, Sade vivant, t. I, Une innocence sauvage, 1740-1777, Paris, Robert Laffont, 1986, p. 94-95.

16. Sade voyait en celle-ci sa principale persécutrice et la seule responsable de sa détention prolongée. À tort, semble-t-il.

17. Sade, « Eugénie de Franval », op. cit., p. 297. 
pas dangereusement toute-puissante. L'écriture permettait la revanche symbolique, elle vient dès lors plutôt recueillir les angoisses, traduisant la hantise du complot ourdi contre soi. Oubliant la manière dont il a dépeint le conseil de Mme de Farneille - «Elle n'avait plus de parents, et pour conseils, que quelques-uns de ces froids amis à qui tout est égal ${ }^{18} \gg-$, Sade le restaure plus loin dans sa puissance grosse de menace - « Furieuse, elle assemble sur le champ son conseil ${ }^{19} \gg-$ et en donne à lire la malveillance insinuante :

[O]n lui fait voir que le meurtre de Valmont va livrer Franval entre ses mains, que le crédit qu'elle redoute va s'éclipser dans un instant, et qu'elle redevient aussitôt maîtresse et de sa fille et d'Eugénie; mais on lui recommande de prévenir l'éclat, et, dans la crainte d'une procédure flétrissante, de solliciter un ordre qui puisse mettre son gendre à couvert ${ }^{20}$.

Derrière ces allusions transparentes aux suites judiciaires de l'affaire de Marseille ${ }^{21}$ se dessine chez l'écrivain le souci d'appréhender un événement - la perte indéfinie de la liberté - dont la compréhension se dérobe à lui, et se trahit le désir de saisir un enchaînement mystérieux de causes : l'écriture se fait ici archéologie, plongée dans un passé que l'on sonde obstinément pour en percer enfin l'opacité intolérable ${ }^{22}$. Mais l'effort entrepris se révèle vain; nulle certitude ne jaillit du cours de la plume, nulle vérité des êtres ne vient s'y dévoiler. Mme de Farneille reste murée dans son ambivalence et se dédouble en deux figures antagonistes : celle de la marâtre machiavélique, à l'affût du moindre faux-pas d'un gendre qu'elle ne songe qu'à perdre et dont elle aigrit les ennemis ${ }^{23}$; celle au contraire de la belle-mère protectrice et dévouée, soucieuse d'épargner à Franval la condamnation qu'il mérite au nom de la défense de l'honneur familial ${ }^{24}$. L'écriture tentait de dissiper l'irritante obscurité des êtres; faute d'y arriver, peut-être, elle en multiplie les contradictions. Ce sont ici ses

18. Ibid., p. 294.

19. Ibid., p. 355.

20. Ibid.

21. L'incarcération de Sade à Vincennes en 1777 se justifiait à l'origine non seulement par la volonté de ne plus lui voir susciter de nouveaux scandales, mais également par le souci de le soustraire à la justice le temps que le jugement d'Aix, qui l'avait condamné à la peine capitale par contumace, soit rejugé.

22. Sade ne cesse de revenir au jugement d'Aix, épisode douloureux qu'il réécrit à diverses reprises dans son œuvre, y faisant notamment allusion dans Les Étrennes philosophiques, Les Cent Vingt Journées de Sodome ou encore Le Président mystifié.

23. « $[R]$ ien de plus certain d'ailleurs, ajoutait-on à Franval, que Mme de Farneille était à la tête des ennemis de son gendre, pour achever de le perdre en le privant de sa liberté, ou en le contraignant à sortir de France, afin de faire incessamment rentrer sous son aile les deux objets chéris qui s'en séparaient» (Sade, « Eugénie de Franval », op. cit., p. 361).

24. « [N]i les sollicitations de Mme de Farneille, ni les démarches de vos parents et de vos amis n'ont pu détourner le glaive de la justice », regrette ainsi Clervil dans les dernières pages de la nouvelle (Ibid., p. 374). 
propres doutes que vient mettre en scène l'écrivain, comme il exprime ailleurs les regrets d'une complicité perdue. L'évocation, dans la bouche de l'épouse, de l'amitié qui aurait pu être entre son mari et sa mère peut du moins se lire en ce sens :

[S]on âme, faite pour aimer, n'a jamais connu l'imposture... tu ne l'apprécias jamais bien, Franval... que ne sus-tu l'aimer comme moi? nous eussions trouvé dans ses bras la félicité sur la terre, c'était l'ange de paix qu'offrait le ciel aux erreurs de ta vie, ton injustice a repoussé son sein, toujours ouvert à ta tendresse, et, par inconséquence ou caprice, par ingratitude ou libertinage, tu t'es volontairement privé de la meilleure et de la plus tendre amie qu'eût créée pour toi la nature ${ }^{25}$.

Elle illustre en tout cas la confusion des sentiments, celle qui, par-delà les éclats de rage et les lettres incendiaires, trouve mieux à se dire dans ce jeu de reflets contradictoires qu'est la littérature.

Présenté complaisamment par le narrateur comme «l'horreur et le miracle de la nature ${ }^{26} »$, le dernier membre de ce quatuor de personnages, Eugénie de Franval, pourrait à première vue passer pour un simple fantasme littéraire, le fruit d'une de ces rêveries incestueuses à laquelle le siècle des Lumières aima tellement à se livrer. Une telle lecture est bien évidemment possible, qui situe la nouvelle dans un héritage de fictions allant de Cleveland (1731-1739) au Mariage de Figaro (1784), de Dom Bougre (1741) à l'Éducation de Laure ${ }^{27}$ (1786). Elle n'interdit pas cependant la nôtre, qui, à la mise en évidence des filiations et des modèles littéraires, préfère la recherche attentive de ce qui dans le texte témoigne d'un investissement autobiographique, plus ou moins conscient, de la part de l'auteur. Suivant cette dernière approche, il semble une nouvelle fois possible - et comme c'était déjà le cas pour les trois autres protagonistes -, de trouver dans la vie de Sade une figure ayant pu servir d'inspiration; car, si Sade n'a jamais vraiment connu sa fille, ni même manifesté le moindre intérêt pour celleci, la liaison mâtinée d'inceste et teintée de scandale qu'il entretint avec la jeune sœur de sa femme, Anne-Prospère de Launay, fait de cette dernière un modèle crédible. Le peu que l'on sait de l'existence de la chanoinesse n'infirme pas cette lecture, bien au contraire. Plus jeune ${ }^{28}$, plus belle et sans doute aussi plus vive intellectuellement que l'épouse légitime, la jeune femme partage certainement plus que l'attrait d'une séduction interdite avec sa doublure romanesque. Le faisceau d'événements qui unit pour un temps sa destinée à celle du marquis confirmerait lui aussi cette hypothèse, tant

25. Ibid., p. 358-359. Cette harmonie des cœurs, c'est dans Aline et Valcour, à travers les personnages de Valcour et de la présidente de Blamont, qu'elle se trouve mise en scène.

26. Ibid., p. 296.

27. On peut d'ailleurs tout à fait lire « Eugénie de Franval » comme la réécriture sadienne de l'œuvre de Mirabeau.

28. Âgée de dix-sept ans au commencement de sa liaison avec le marquis, qui en comptait vingt-neuf, Anne-Prospère aurait d'ailleurs presque eu l'âge d'être la fille de celui-ci. 
le cours de leurs existences respectives semble guider celui d' « Eugénie de Franval ». Au-delà de la ressemblance des acteurs, c'est en effet la structure même de l'intrigue qui reproduit les étapes de ce qu'on considère généralement comme la grande histoire d'amour du marquis. La période de bonheur goûté par Franval et par sa maîtresse ravive très certainement le souvenir d'un bonheur perdu, celui des premiers temps d'une liaison jusqu'à récemment très mal connue. Longtemps réduite par les biographes à l'embrasement fugace de deux cœurs au creux de l'année 1772, l'idylle d'Anne-Prospère et de Sade, éclairée par de nouveaux documents ${ }^{29}$, semble en définitive couvrir une période bien plus longue et remonter à l'année 1769. La singulière indulgence de Mme de Franval pour sa fille après leur réconciliation en trompe-l'œil, pourrait dans ce contexte faire écho à celle de Renée-Pélagie, qui semble avoir fermé les yeux sur la liaison de sa sœur avec son époux. La fuite de Franval traqué par la justice après le meurtre de Valmont ne peut que rappeler celle du couple scandaleux vers l'Italie à l'été 1772, à la suite des remous suscités par l'affaire de Marseille. Le meurtre de grand chemin qui perd le héros de la fiction est certainement moins infamant que les accusations d'empoisonnement et de sodomie dont fit l'objet l'écrivain maudit, mais le résultat final en est le même : Franval se trouve condamné à perdre la tête, Sade est exécuté en effigie; un coup du sort vient abattre le libertin « ruiné $^{30}$ " par la confiscation de ses biens et « à jamais flétri ${ }^{31}$ », la même mort sociale et économique frappe Sade à petit feu dans les années qui suivent le jugement d'Aix. Si, dans la nouvelle comme dans l'existence réelle, la liaison défendue trouve son châtiment dans la déchéance du héros, l'écriture offre à la passion des amants une pureté dans le crime qui n'est pas celle des amours envolées de Sade : Franval se fixait dans une passion exclusive, les infidélités de Sade finissent par lui faire perdre Anne-Prospère; le suicide sanglant du premier théâtralise la sortie d'un héros du mal, la tentative identique, mais manquée ${ }^{32}$, de Donatien lui refuse l'éclat d'un destin tragique. Dans les deux cas par contre, c'est la femme la première qui se retire du jeu : prise d'un remords subit, gagnée finalement par la nature, Eugénie se consume et meurt sur le sein de sa mère; lassée des infidélités de son amant volage, Anne-Prospère le quitte et part mourir au couvent quelques années plus tard.

29. Voir l'exceptionnel document édité par Maurice Lever, « Je jure au marquis de Sade, mon amant, de n'être jamais qu'à lui... ", Paris, Fayard, 2005, qui confirme et complète les brillantes intuitions présentées par Jean-Jacques Pauvert dans la biographie qu'il consacre à l'écrivain (op. cit., p. 241-242).

30. Sade, «Eugénie de Franval », op. cit., p. 374.

31. Ibid.

32. Si dans la fiction la mort édifiante de Franval est largement diffusée par l'abbé de Clervil, la tentative ratée de Sade sera par contre prudemment étouffée par sa famille, au point de ne parvenir à la connaissance des biographes que ces dernières années. Voir Maurice Lever, «Je jure au marquis de Sade... », op. cit., p. 49-57. 
Sous la plume de Sade, le souvenir amoureux se hisse donc à la hauteur du mythe, les écarts d'un tempérament ardent se font crimes de l'amour ${ }^{33}$. L'écriture de soi ne se borne pas cependant à transmuer le vécu en archétype, mais s'attache également à procurer des jouissances symboliques ${ }^{34}$. À lire tous les petits écarts qui viennent distinguer Franval de son modèle, ces subtiles différenciations par lesquelles la fiction semble prendre son autonomie de la vie réelle, il est frappant de voir à quel point Sade semble soucieux de se projeter en majesté dans sa fiction; comme si la mise en scène de soi pouvait réparer les torts de la vie, effacer les humiliations subies, rétablir un ordre des choses. Cette dimension compensatrice, ce rééquilibrage via les prestiges de l'imagination trouvait à s'exprimer, comme on l'a vu, dans l'inversion des situations financières des personnages - la mésalliance subie s'effaçait dans le libre choix d'une épouse vouée à la soumission par son infériorité socio-économique - comme dans celle des rôles d'emprisonneur et d'emprisonné35. Elle peut sans doute se résumer à la quête fantasmée d'une maîtrise totale des choses et des êtres. Si Sade ressentit durement tout au long de sa vie le pouvoir d'autrui sur sa destinée, son Franval apparaît tout au contraire comme celui qui distribue les rôles : l'écrivain avait souffert en prison d'accès de jalousie délirante à l'égard de sa femme, il imagine à l'inverse un mari tentant d'orchestrer la séduction de la sienne par un tiers; indéfectiblement vertueuse, celle-ci maintient une inébranlable fidélité à son époux. L'écriture offre ainsi à l'imagination inquiète la mise en scène rassurante d'une maîtrise des événements, le réconfort d'un amour indéfectiblement maintenu par-delà sa mise à l'épreuve. Elle lui permet surtout de liquider ce qui semble être une angoisse fondamentale de l'écrivain : l'inquiétante liaison de la mère et de la fille, cette part obscure de l'amante qui échappe à son amant, cet indéfinissable secret féminin sur lequel achoppe le pouvoir de celui-ci.

«Eugénie de Franval» se place tout entier sous le signe de cette obsession, articulant à l'expérience philosophique d'une dénaturation réussie - destruction du lien mère/fille - la mise en scène d'une victoire de l'amant sur sa belle-mère. Dans la nouvelle, Mme de Farneille apparaît en effet comme une figure possessive, une rivale dangereuse dans la lutte pour l'appropriation des femmes : elle fait enlever Eugénie, rappelle à elle sa fille partie accompagner Franval dans son exil et ne semble avoir pas

33. Sade, ne l'oublions pas, est hanté par un mythe familial de la passion, celui des amours supposées de Pétrarque et de sa propre ancêtre Laure. Le Projet de mausolée envoyé depuis Miolans à l'automne 1772 à Anne-Prospère peut se lire comme la trace du désir précoce de donner à sa passion défunte une aura mythique : «Ci-git, immolé par l'amour, / Celui dont il fit sa victime. / On sut, en le privant du jour, / Sur lui seul venger tout le crime " (Ibid., p. 58, nous soulignons). Il préfigure en cela « Eugénie de Franval ».

34. La même qu'il éprouve par exemple à ridiculiser ses juges dans Le Président mystifié. 35. Aux fantasmes d'emprisonnement déjà cités s'ajoute l'épisode de la séquestration de M. de Clervil. 
d'autre motivation dans sa lutte contre son gendre que celle de récupérer ces deux objets chers à son cœur ${ }^{36}$. Le libertin doit parer chacun de ses coups pour conserver la possession de ce qui lui « appartient ${ }^{37} »$ : il en neutralise les séides - Clervil -, assassine les alliés - Valmont - et dénonce les visées malveillantes. Son succès se mesure alors dans l'emprise amoureuse qu'il parvient à préserver sur son épouse : la docilité de celle-ci, qui se résout à la séparation d'avec sa mère, son obéissance jusque dans la mort - refus de rentrer à Paris malgré les injonctions de Mme de Farneille - entérinent la victoire du libertin sur sa rivale. De même, le matricide final commis par Eugénie fait figure, à l'échelon générationnel suivant, d'aboutissement du long travail de sape du lien filial entrepris par Franval : mise en demeure de choisir entre un père-amant qu'elle idolâtre et une mère dont on a pris soin de la tenir toujours écartée, la jeune fille prouve, comme attendu, la vérité de son amour dans l'accomplissement du plus criminel des actes. En cela, la littérature accomplit symboliquement ce que l'existence a refusé au marquis : le don total de soi de la part de la femme aimée, son rejet de toute autre loi que celle du libertin. Sade a connu l'amertume d'une séparation, le lent détachement d'une épouse au fil de trop longues années de prison: ses lettres à sa femme ne cessent de la mettre en garde contre les noires manigances qu'il prête à sa belle-mère, scrutent avec inquiétude la possibilité d'un rapprochement mère-fille, tentent de conserver RenéePélagie de son côté à toute force. Imprécations, plaintes et cajoleries n'y suffisent pourtant pas : les dernières années de détention du marquis voient se creuser un fossé entre lui et son épouse; sa libération achève de les séparer. Sade lira dans cette rupture aussi bien l'œuvre des confesseurs que l'ultime vengeance de la présidente de Montreuil. On tient sans doute ici l'une des explications de cette hostilité viscérale à la mère qui parcourt l'ensemble de ses écrits ${ }^{38}$.

À lire « Eugénie de Franval », il ne fait guère de doute à qui connaît la vie du marquis de Sade qu'il se trouve là devant une œuvre en partie autobiographique. La silhouette des acteurs, la succession des événements, tout vient trop faire écho à ce que l'on sait de son existence pour ne voir là qu'un simple hasard de plume, un jeu de ressemblances fortuites. Mais l'écrivain se masque : à la transparence des cœurs et des âmes - celle qui se cherche et se célèbre au même moment dans Les Confessions de Rousseau -, il préfère l'allusion cryptée, les gazes de la fiction. Si les dévoilements

36. Voir les passages cités correspondant aux notes 20 et 23.

37. Sade, « Eugénie de Franval », op. cit., p. 295.

38. On a beaucoup glosé sur l'origine de cette haine sadienne de la mère. Il nous semble pour notre part fort clair qu'elle renvoie à la détestation de celle qui fut pour Sade aussi bien une mère de substitution qu'une belle-mère intrusive. Détestation qui n'était pourtant pas sans nuance, comme on l'a vu. Même analyse, à peu de choses près, chez Maurice Lever dans son Donatien Alphonse François, marquis de Sade, Paris, Fayard, 1991, p. 122. 
sadiens sont toujours partiels, placés sous le signe de l'indirect, ils n'en offrent pas moins à qui sait lire un réseau précieux d'indices et d'aveux, car la littérature est cet espace où, dans le libre jeu du fantasme, l'auteur se réinvente et se trahit; où viennent se dire ses nostalgies et ses rancœurs, se dessinent ses regrets et se prennent ses revanches. Déplacement, inversion, contradiction sont les lois de cette écriture de soi qui ne se borne pas à prendre en charge ce qui a été, mais s'ouvre aussi à ce qui aurait pu ou dû être, à ce qui refuse de se laisser oublier, à ce à quoi, enfin, l'on ne peut s'empêcher de revenir. Ces logiques sous-jacentes qui viennent travailler l'écriture, la nouvelle les laisse affleurer plus qu'aucun autre écrit de Sade. Il ne faudrait pas croire, pourtant, que le reste de l'œuvre y échappe : d'une page d'un texte à celle d'un autre se retrouvent en effet les mêmes motifs, se donnent à lire les mêmes rêveries obsessionnelles; seuls les distinguent alors l'infini jeu des variations, l'art subtil des nuances. Les biographèmes, ces atomes d'existence, ne cessent de se réagencer et se recomposer d'une œuvre à l'autre, affirmant l'ambivalence des sentiments comme la confusion des élans. Leur modulation incessante interroge le passé, tente de lever l'obscurité des êtres: Sade en prison traquait obsessionnellement les « signaux » de ses proches, l'écriture n'est pour lui, peut-être, que la poursuite de cette entreprise de déchiffrement, mais par d'autres moyens.

Vincent Jolivet

Université Paris-Sorbonne, CELLF $17^{e}-18^{e}$ 